\title{
Effect of Distant Sidewalls on Wave-Number Selection in Rayleigh-Bénard Convection
}

\author{
M. C. Cross \\ Bell Laboratories, Murray Hill, New Jersey 07974 \\ and \\ P. G. Daniels \\ Department of Mathematics, The City University, London, England \\ and
}

P. C. Hohenberg

Bell Laboratories, Murray Hill, New Jersey 07974

and

E. D. Siggia

Laboratory of Atomic and Solid State Physics, Cornell University, Ithaca, New York 14853

(Received 10 July 1980)

\begin{abstract}
An analysis is presented of the steady states of two-dimensional convection in a laterally finite rectangular container near threshold. It is shown that the presence of sidewalls severely restricts the allowed wave vectors which can occur in the bulk of the container. This effect provides a possible mechanism to explain the observed wavelength increase of convective rolls with increasing Rayleigh numbers.
\end{abstract}

PACS numbers: 47.25.Qv, 03.40.Ge, 47.10.+ g

There exists at present no adequate theoretical understanding of the phenomenon of wave number selection in cellular flows such as Rayleigh-Bénard convection. ${ }^{1-4}$ As a step along the way to such an understanding, we have analyzed the steady states of two-dimensional convection immediately above threshold, in a finite rectangular container with large aspect ratio (ratio of width to height, $2 L \gg 1$ ). We find that the presence of rigid sidewalls imposes severe restrictions on the possible wave vectors which can occur in the bulk of the container. Specifically, at Rayleigh number $R$, the band of allowed wave vectors $\overrightarrow{\mathrm{k}}=\overrightarrow{\mathrm{q}}$ $+\overrightarrow{\mathrm{q}}_{0}$, about the critical wave vector $\overrightarrow{\mathrm{q}}_{0}$, is reduced from a size $|\overrightarrow{\mathrm{q}}| \sim\left[\left(R-R_{0}\right) / R_{0}\right]^{1 / 2}$ in the laterally infinite system, to the range $|\overrightarrow{\mathrm{q}}| \sim\left(R-R_{0}\right) / R_{0}$ in the presence of rigid sidewalls $\left(R_{0}\right.$ is the critical Rayleigh number of the infinite system). The precise range depends on both the Prandtl number $\sigma$ of the fluid and the thermal properties of the sidewalls in a way which we calculate to lowest order in $\left(R-R_{0}\right) / R_{0}$.

Our starting system is represented by the twodimensional Oberbeck-Boussinesq equations ${ }^{2,3}$ for a layer of fluid confined between horizontal plates, at which for mathematical convenience we assume free boundary conditions. ${ }^{2,3}$ At the sidewalls, on the other hand, it is essential that we impose rigid boundary conditions on the flow velocities $(\overrightarrow{\mathrm{u}}=0)$. The thermal boundary condition is expressed in terms of a parameter $\mu$, proportional to the conductance of the sidewall.

Near threshold, where the effects of nonlinearity are weak, the hydrodynamic equations may be consistently expanded in a power series in the small parameter $\epsilon^{1 / 2}$, where $\epsilon \equiv\left(R-R_{0}\right) / 18 \pi^{2}$. Specifically, the stream function $\psi(x, z)$ (whose derivatives yield the velocities) is written in the form $^{5,6}$

$$
\psi(x, z)=i(4 / \pi) \epsilon^{1 / 2}\left[A_{0}(X) \exp \left(i q_{0} x\right)-A_{0} *(X) \exp \left(-i q_{0} x\right)\right] \sin \pi z+O(\epsilon),
$$

in terms of the amplitude function $A_{0}(X)$ which varies on the slow scale $X \equiv x \epsilon^{1 / 2}$ [for our case of free horizontal boundary conditions $R_{0}=27 \pi^{4} / 4$ and $q_{0}=\pi / \sqrt{2}$, where the usual ${ }^{6}$ dimensionless units are used throughout]. An expression similiar to (1) holds for the temperature deviation $T(x, z)$. If the expansion is carried to sufficiently high order in $\epsilon^{1 / 2}$ one obtains an amplitude equa- tion in steady state, of the form ${ }^{7,8}$

$$
A^{\prime \prime}+A-|A|^{2} A+\epsilon^{1 / 2} F_{1 / 2}[A]+O(\epsilon)=0,
$$

where $A(X)=A_{0}(X)+\epsilon^{1 / 2} A_{1}(X)+\cdots$, and $F_{1 / 2}[A]$ is a functional of $A$ and its $X$ derivatives, $A^{\prime}, A^{\prime \prime}$, ... . To lowest order in $\epsilon^{1 / 2}$, Eq. (2) reproduces the well-known amplitude equation of Refs. 5 and 
6.

For the laterally finite system it may be shown by matching to the linearized hydrodynamic equations near the walls that the appropriate boundary conditions for (2) are $\mathrm{ar}^{7,8}$

$$
\begin{aligned}
& A-\epsilon^{1 / 2} \alpha_{ \pm} A^{\prime}-\epsilon^{1 / 2} \beta_{ \pm} A^{* \prime}=O(\epsilon), \\
& X= \pm \delta= \pm L \epsilon^{1 / 2},
\end{aligned}
$$

where $\alpha_{+}=-\alpha_{-}{ }^{*}=\alpha, \beta_{+}=-\beta_{-}^{*}=\beta$, and $\alpha, \beta$ are specified complex numbers of order unity which depend on $\mu$.

Since a detailed analysis of the solution of (2) and (3) is rather complicated, we shall illustrate the mechanism for restricting the allowed band of wave vectors on the basis of a number of simpler phenomenological models. The detailed analysis of (2) and (3) will be presented elsewhere, ${ }^{7}$ and only the main results will be summarized here. The simplest model consists of the lowestorder equation

$$
A^{\prime \prime}+A-|A|^{2} A=0
$$

with periodic boundary conditions $A(X=\delta)=A(X$ $=-\delta)$. This system has "phase-winding" solutions

$$
A=\left(1-Q^{2}\right)^{1 / 2} \exp (i Q X),
$$

with $Q=n \pi / \delta, n=0, \pm 1 \ldots,|n| \leqslant \delta / \pi$. In terms of the physical scale $x$, the wave vector is $q \equiv Q \epsilon^{1 / 2}$ $=n \pi / L$, with $-\epsilon^{1 / 2} \leqslant q \leqslant \epsilon^{1 / 2}$. Thus the only effect of the sidewalls is to quantize $q$, but not to restrict the band of allowed values.

The solutions of (2) which match the linearized hydrodynamic equations near the sidewalls are those for which the amplitude $A$ at $X= \pm \delta$ is reduced to $O\left(\epsilon^{1 / 2}\right)$ by the boundary conditions (3). It is thus instructive to study a phenomenological model (I) consisting of Eq. (4), with the boundary condition

$$
A(X)=\sqrt{2 \lambda} \exp \left( \pm i \theta_{0}\right), \quad X= \pm \delta= \pm L \epsilon^{1 / 2},
$$

with $\lambda$ and $\theta_{0}$ real, and we suppose $\lambda \ll 1$. Let us rewrite Eq. (4) in terms of phase and magnitude variables $A(X) \equiv r(X) \exp [i \theta(X)]$ as $^{6}$

$$
\begin{aligned}
& r^{2} \theta^{\prime}=Q, \\
& \frac{1}{2} r^{\prime 2}+Q^{2} / 2 r^{2}+\frac{1}{2} r^{2}-\frac{1}{4} r^{4}=E,
\end{aligned}
$$

where $Q$ and $E$ are real constants. From (7) and (8) it may be shown ${ }^{7}$ that if the magnitude $r$ is decreased to a value $r_{m}$ anywhere, then

$$
|Q|<\left(r_{m} / \sqrt{2}\right)\left(1-r_{m}^{2} / 2\right) .
$$

It will turn out that $Q$ plays the role of the wave number in the bulk of the container, as in (5). Thus, the depression of the magnitude $r$ to a small value $\sqrt{2} \lambda \ll 1$ at the sidewalls [Eq. (6)] provides the mechanism for restricting the wave numbers, via $\mathrm{Eq}$. (9). Indeed, it may be shown ${ }^{7}$ that phase-winding solutions, having the form (5) in the bulk, exist for model I. Integrating Eq. (7) between the boundaries at $X= \pm \delta$, and evaluating the contributions from the bulk and boundary regions leads to two classes of solutions defined by the conditions

(i) $Q= \pm \lambda \sin \left(Q \delta-\theta_{0}\right)$,

or

(ii) $Q_{n} \delta=\theta_{0}+\left(n+\frac{1}{2}\right) \pi ;\left|Q_{n}\right|<\lambda$.

The solutions ${ }^{7}$ of (10) consist of a set of wave vectors in the reduced range $|q| \leqslant \lambda \epsilon^{1 / 2}$, roughly quantized in units of $\pi / L$.

Another phenomenological model, which is closer to our starting system (2) and (3), consists in dropping the term $F_{1 / 2}$ in (2) [i.e., replacing (2) by (4)], but keeping the boundary condition (3) up to $O\left(\epsilon^{1 / 2}\right)$ (model II). For this model it may again be shown ${ }^{7}$ that phase-winding solutions exist, with $A^{\prime}$ of order unity and $A=O\left(\epsilon^{1 / 2}\right)$ at the boundary. The conditions analogous to (10) are

$$
\begin{aligned}
& \text { (i) } Q=\frac{1}{2} \epsilon^{1 / 2}\left[-\alpha_{i} \pm|\beta| \sin \left(2 Q \delta-\varphi_{\beta}\right)\right], \\
& \text { (ii) } 2 Q_{n} \delta=\varphi_{\beta}+\left(n+\frac{1}{2}\right) \pi ; \\
& \qquad\left|2 Q_{n}+\epsilon^{1 / 2} \alpha_{i}\right|<\epsilon^{1 / 2}|\beta|,
\end{aligned}
$$

where $\alpha_{i}=\operatorname{Im} \alpha$ and $\varphi_{\beta}$ is the phase of $\beta$. The important difference between (10) and (11) is that the solution of (11) consists of an asymmetric band of wave vectors $q_{-} \leqslant q \leqslant q_{+}$, with $q_{ \pm}=\frac{1}{2} \epsilon$ $\left(-\alpha_{i} \pm|\beta|\right)$.

For the starting system (2)-(3), which corresponds to realistic sidewall boundary conditions it turns out ${ }^{7}$ that the effect of the $\operatorname{term} F_{1 / 2}[A]$ in (2) is merely to change the parameter $\alpha_{i}$ in (11) to $\alpha_{i}=\alpha_{i}-b(\sigma)$, where $b(\sigma)$ is a real function of the Prandtl number $\sigma$. The allowed band of wave vectors is again linear in $\epsilon$ and asymmetric, with

$$
q_{ \pm}=(|\beta| / 2)(-\eta \pm 1) \epsilon .
$$

Our calculation ${ }^{7}$ yields

$$
\begin{aligned}
\eta=(32 \sqrt{3})^{-1}\left(5+21 \sigma^{-1}\right. & \left.+40 \sigma^{-2}\right) \\
& \times\left(1+4 \bar{\mu}+6 \bar{\mu}^{2}\right)^{-1 / 2},
\end{aligned}
$$

with $\bar{\mu}=(1+2 \mu / \pi)^{-1}$. Equation (12) then implies that $q_{-}<0$ for all $\sigma$ and $\mu$, whereas $q_{+}$can be ei- 


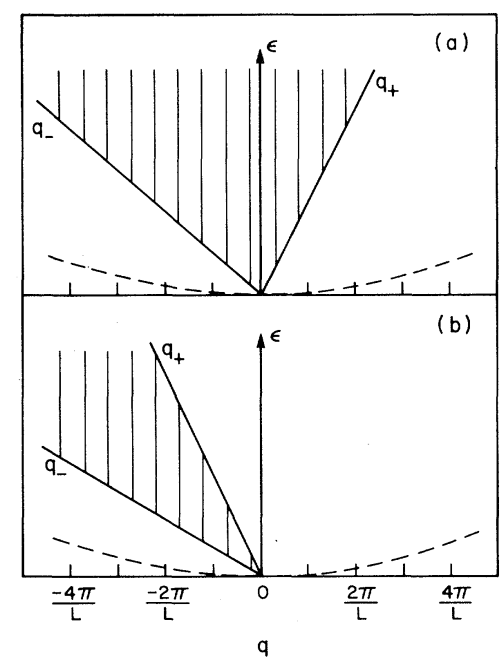

FIG. 1. Schematic diagram of the allowed states of convection as a function of reduced Rayleigh number $(\epsilon)$ and wave vector $\left(\overrightarrow{\mathrm{q}}=\overrightarrow{\mathrm{k}}-\overrightarrow{\mathrm{q}}_{0}\right)$, for a container of length $2 L$. For simplicity only the solutions (ii) in (11) are shown. The Rayleigh number dependence of (i) is more complicated. In case (a) $\left(q_{+}>0\right), q$ changes little with increasing $\epsilon$. For case (b) $\left(q_{+}<0\right)$ q must decrease discontinuously as $\epsilon$ increases. The dashed line is the lower limit for convective states in the infinite system.

ther positive or negative. For $\sigma \gg 1$ we have $q_{+}$ $>0$ [Fig. 1(a)], and the states of given $q$ evolve smoothly as a function of $\epsilon$. In the opposite case $(\sigma \ll 1)$ where $q_{+}<0$ [Fig. $1(\mathrm{~b})$ ], each state has both a minimum and a maximum $\epsilon$, and the system shifts discontinuously from one wave vector to the other as $\epsilon$ is raised or lowered. This corresponds to the disappearance or creation of a roll at the boundary of the container. Note that the limiting values $q_{t}$ are independent of the aspect ratio $L$, and also apply to the semi-infinite case, where only one boundary is present. For a physical system of size $L$, the time necessary for the boundaries to influence the steady state in the bulk will grow as $L$ becomes large.

The above calculation thus provides a mechanism for restricting the band of available wave vectors of steady convection, coming solely from the sidewall boundary conditions. Because of the idealized nature of our starting equations the results cannot be used directly to explain any experimental findings, but the effects found here must surely enter into a more realistic theory. We shall conclude making some qualitative remarks concerning such a theory.

(i) Our calculations are for free-free horizontal boundary conditions. The rigid case is analytically much more difficult, but we expect similar ef- fects to occur there, since an amplitude equation also exists for this case. ${ }^{9}$ The values of $q_{ \pm}$as a function of $\sigma$ and $\mu$ are of course expected to be different from those found here. On the basis of experiment ${ }^{1}$ we might speculate that $q_{+}<0$ for most values of $\sigma$. Alternatively, in the Taylor problem, ${ }^{10}$ it appears that $q_{+}>0$.

(ii) The present work calculates steady solutions of the equations without discussing the much more difficult question of stability. ${ }^{2-6}$ We may remark that for the infinite system the band of stable wave vectors for two-dimensional motion is $-\epsilon^{1 / 2} / \sqrt{3} \leqslant q \leqslant \epsilon^{1 / 2} / \sqrt{3}$, which space the domain (12). The possibility of additional instabilities in the finite system cannot be ruled out, however, and it is presently under investigation.

(iii) The influence of the direction transverse to $x$ (three-dimensional motion) has been discussed in the infinite system, where the most important effect near threshold is the zigzag instability, ${ }^{3,6}$ which for free-free horizontal boundary conditions eliminates ${ }^{2}$ all states with $q<-c_{0} \epsilon^{2}\left[c_{0}\right.$ $=O(1)]$. It is thus interesting that for small $\sigma$ we find $q_{+} / \epsilon<0$, i.e., all the states of steady two-dimensional flow of the finite system are in the band of unstable wave vectors of the infinite case. Note, however, that the zigzag instability may itself be modified by the finite size in the transverse direction.

(iv) The expansion leading to (2) is based on the smallness of the term $\epsilon^{1 / 2} F_{1 / 2}$, which is proportional to $\epsilon^{1 / 2} / \sigma$ for $\sigma \rightarrow 0$. Thus the domain of validity of our expansion shrinks to zero in that limit. $^{3}$

(v) Experiments are often performed in cylindrical containers, ${ }^{1}$ where an additional singularity occurs in the center of the cell. ${ }^{11}$ It would be interesting to extend our analysis to that case, but in the absence of such a calculation we might speculate that the cylinder should show similar restrictions on the allowed band of $q$.

(vi) We have repeated the present analysis for the two models introduced in Ref. 4, and find that near threshold they are special cases of Eqs. (2) and (3) above, with particular values of $\alpha, \beta$, and $F_{1 / 2}$. The limiting wave vectors of Eq. (12) turn out to be $q_{ \pm}= \pm\left(16 q_{0}^{3}\right)^{-1} \epsilon$ [model (a)] and $q_{+}$ $=-\left(\frac{47}{48} q_{0}{ }^{3}\right) \epsilon, q_{-}=-\left(\frac{53}{48} q_{0}{ }^{3}\right) \epsilon[$ model (b)], which appears to be consistent with the numerical calculations ${ }^{4}$ Note that Ref. 4 treats the important question of dynamics and stability, about which we cannot at present say anything.

In conclusion, our analysis has shown that finite sidewalls restrict the band of allowed wave 
vectors for steady two-dimensional convection, in a manner which depends on the Prandtl number and the thermal properties of the sidewalls. This effect provides a possible mechanism for understanding the phenomenon of wave number selection. An interesting consequence of our calculations, which could be tested experimentally is the distinction between the case represented in Fig. 1(a), where a single wave vector remains as $\epsilon$ grows, and the case of Fig. 1(b) where the wave vector must change discontinuously with increasing $\epsilon$.

The authors wish to acknowledge the hospitality of the Aspen Center for Physics, where this work was begun. One of us (P.G.D.) also thanks Bell Laboratories for hospitality. This research was partially supported by the National Science Foundation under Grant No. DMR-77-18329.
${ }^{1}$ E. L. Koschmieder and S. G. Pallas, Int. J. Heat Mass Transfer 17, 991 (1974).

${ }^{2}$ D. D. Joseph, Stability of Fluid Motion (Springer, New York, 1976), Chap. 11.

${ }^{3}$ F. H. Busse, Rep. Prog. Phys. 41, 1929 (1978).

${ }^{4}$ Y. Pomeau and P. Manneville, Phys. Lett. 75A, 296 (1980).

${ }^{5}$ L. A. Segel, J. Fluid Mech. 38, 203 (1969).

${ }^{6}$ A. C. Newell and J. A. Whitehead, J. Fluid Mech. $\underline{38}$, 279 (1969).

${ }^{7}$ M. C. Cross, P. G. Daniels, P. C. Hohenberg, and E. D. Siggia, to be published.

${ }^{8}$ P. G. Daniels, Proc. Roy. Soc. London, Ser. A 358 , 173 (1977), and Mathematika $\underline{25}, 216$ (1978).

${ }^{9}$ M. C. Cross, to be published; R. E. Kelly and D. Pal, J. Fluid Mech. 86, 433 (1978).

${ }^{10}$ E. L. Koschmieder and J. Burkhalter, J. Fluid Mech. 58, 547 (1973)

${ }^{11}$ S. N. Brown and K. Stewartson, Proc. Roy Soc. London, Ser. A $\underline{360}, 455$ (1978). 